\title{
Direct cadaverine production from cellobiose using $\beta$-glucosidase displaying Escherichia coli
}

\author{
Naoki Ikeda, Mari Miyamoto, Noriko Adachi, Mariko Nakano, Tsutomu Tanaka and Akihiko Kondo*
}

\begin{abstract}
In this study, we demonstrate the one-step production of cadaverine (1,5-diaminopentane) from cellobiose using an Escherichia coli strain displaying $\beta$-glucosidase (BGL) on its cell surface. L-lysine decarboxylase (CadA) derived from E. coli and BGL from Thermobifida fusca YX (Tfu0937) fused to the anchor protein Blc from E. coli were co-expressed using E. coli as a host. The expression of CadA was confirmed by Western blotting and BGL activity on the cell surface was evaluated using PNPG as a substrate. Growth on cellobiose as the sole carbon source was also achieved. The OD600 value of the BGL and CadA co-expressing strain was 8.0 after $48 \mathrm{~h}$ cultivation, which is higher than that obtained by growth on glucose ( 5.4 after $48 \mathrm{~h}$ cultivation). The engineered strain produced cadaverine from cellobiose more effectively than from glucose: $6.1 \mathrm{mM}$ after $48 \mathrm{~h}$ from $28 \mathrm{~g} / \mathrm{L}$ of consumed cellobiose, vs. $3.3 \mathrm{mM}$ from $20 \mathrm{~g} / \mathrm{L}$ of consumed glucose.
\end{abstract}

Keywords: Cadaverine; E. coli; Cell surface display; Cellobiose

\section{Introduction}

Cadaverine, also known as 1,5-diaminopentane, has many industrial applications. Similar to putrescine (1,4-diaminobutane), cadaverine serves as a component of polymers such as polyamides and polyurethane, and as a component of chelating agents and other additives. Cadaverine is particularly relevant in the production of bio-polyamides derived from renewable feedstocks as a replacement for conventional polyamides derived from petrochemicals. The petrochemical route suffers from the limited supply and rising price of fossil fuels, as well as from low eco-efficiency. Polyamide 54 is made by the polycondensation of cadaverine and succinic acid. Polyamide 54 holds promise as a bio-based alternative to conventional petroleum-based polyamides that are currently produced on the order of 3.5 million tons annually (Kind et al. 2010; Mimitsuka et al. 2007).

In the Gram-negative bacterium Escherichia coli, cadaverine is biosynthesized directly from L-lysine by two kinds of L-lysine decarboxylases: a constitutive one encoded by the $l d c C$ gene, and an inducible one produced at low $\mathrm{pH}$ encoded by the $\operatorname{cad} A$ gene. The level of cadaverine in $E$. coli is regulated by biosynthesis, degradation, uptake, and export (Soksawatmaekhin et al. 2004).

\footnotetext{
* Correspondence: akondo@kobe-u.ac.jp

Department of Chemical Science and Engineering, Graduate School of Engineering, Kobe University, 1-1 Rokkodai-cho, Nada, Kobe 657-8501, Japan
}

It was recently shown that E. coli can tolerate a higher concentration of cadaverine, just slightly lower than that tolerated by a wild-type Corynebacterium glutamicum strain (Mimitsuka et al. 2007; Qian et al. 2011). This high tolerance suggests that microorganisms can be metabolically engineered to overproduce cadaverine to industrially useful levels.

E. coli is an attractive bacterium due to its fast growth in inexpensive media, demonstrated scale-up processes, well-understood genetic background and metabolism, and the availability of established metabolic engineering tools. In particular, this bacterium has recently been metabolically engineered to produce biomass-derived biofuels and chemicals (Atsumi et al. 2008; Lee et al. 2007; Park et al. 2008; Park et al. 2007; Qian et al. 2009; Steen et al. 2010; Tyo et al. 2009). A cadaverine overproducing $E$. coli strain has been engineered by the introduction of the cadaverine biosynthesis, degradation, and utilization pathways (Na et al. 2013; Qian et al. 2011). This metabolically engineered $E$. coli strain could constitutively produce cadaverine to a high concentration in glucose mineral salts medium. However, there are no reports of the use of E. coli strain to produce cadaverine using cellobiose as a carbon source.

Lignocellulosic biomass is one of the most attractive bioresources because of its abundance and the fact that it 
is renewable. The use of lignocellulosic biomass has therefore recently garnered attention (Sánchez and Cardona 2008). However, in order to use lignocellulose as a bioresource, costly and complex intermediate chemical steps such as milling and hydrolysis treatment, followed by fermentation, are required (Sánchez and Cardona 2008). It is therefore important to develop an efficient and costeffective method to convert lignocellulosic biomass into simple fermentable sugars.

The efficient degradation of cellulose requires a synergistic reaction of the cellulolytic enzymes endoglucanase (EG), cellobiohydrolase (CBH), and $\beta$-glucosidase (BGL). The cellulose is degraded by EG and $\mathrm{CBH}$, resulting in cellobiose and some cellooligosaccharides, which can be converted to glucose by BGL. BGL catalyzes the final step in cellulose degradation, as well as stimulating cellulose hydrolysis by relieving the cellobiose-mediated inhibition of EG and CBH. To date, there have been only a few reports on the production of valuable chemicals such as isopropanol (Soma et al. 2012) from cellobiose or cello-oligosaccharides by directly using $E$. coli, and no reports of the production of cadaverine from cellobiose using E. coli. The use of BGL-expressing E. coli may lead to reduced usage of cellulases, thereby reducing the significant costs of hydrolysis in the production of cellulosic biomass-derived products.

Here, we demonstrate direct cadaverine production using cellobiose as a carbon source. We employed cell surface display, which is a powerful tool for improving the activity of a displayed protein (Lee et al. 2003; Tanaka et al. 2011). We previously reported a cell surface display system in E. coli using Blc as an anchor (Tanaka et al. 2011) and the direct assimilation of cellobiose using defined minimum medium. In the present study, using $E$. coli as a host, we demonstrate the direct production of cadaverine from cellobiose.

\section{Materials and methods}

\section{Bacterial strains and media}

Table 1 shows the strains and plasmids used in this study. E. coli JCM20137 was used as the host strain. This bacterium, and its derivative possessing a plasmid, were grown in Luria-Bertani medium (10 g/l tryptone, $5 \mathrm{~g} / \mathrm{l}$ yeast extract, $5 \mathrm{~g} / \mathrm{l}$ sodium chloride) containing $100 \mu \mathrm{g} / \mathrm{ml}$ ampicillin at $37^{\circ} \mathrm{C}$. For cadaverine fermentation, $\mathrm{R} / 2$ (Qian et al. 2011) medium containing $100 \mu \mathrm{g} / \mathrm{ml}$ ampicillin and $40 \mathrm{~g} / \mathrm{l}$ glucose or $40 \mathrm{~g} / \mathrm{l}$ cellobiose was used. $\mathrm{R} / 2$ medium ( $\mathrm{pH} 6.80$ ) contains $3.17 \mathrm{~g} / \mathrm{l}$ of $\mathrm{NaNH}_{4} \mathrm{HPO}_{4} 4 \mathrm{H}_{2} \mathrm{O}, 6.75 \mathrm{~g} / \mathrm{l}$ of $\mathrm{KH}_{2} \mathrm{PO}_{4}$, $0.85 \mathrm{~g} / \mathrm{l}$ of citric acid, $0.7 \mathrm{~g} / \mathrm{l}$ of $\mathrm{MgSO}_{4} 7 \mathrm{H}_{2} \mathrm{O}$ and $5 \mathrm{ml} / \mathrm{l}$ of a trace metal stock solution (Lee and Chang 1993).

\section{Plasmid construction}

Oligonucleotide primers used for plasmid construction are listed in Table 1. Polymerase chain reaction (PCR) was conducted using KOD FX DNA polymerase (TOYOBO Co., Ltd., Osaka, Japan).

The plasmid for lysine decarboxylase (CadA) expression was constructed as follows. The gene encoding CadA was amplified by PCR using CadA_F and CadA-FLAG_R primers with $E$. coli Novablue genomic DNA as the template. The amplified fragment was introduced into the BglII and SalI sites of plasmid pHLA (Narita et al. 2006). The resulting plasmid was designated pHLA-cadA. The plasmid for both CadA and BGL expression was constructed as follows. The gene encoding the HCE promoter BlcTfu0937 terminator region was amplified by PCR using HCE-blc-Tfu-term_F and HCE-blc-Tfu-term_R primers with plasmid pHLA-blc-Tfu (Tanaka et al. 2011) as a template. The amplified fragment was ligated into plasmid pHLA-cadA using an In-fusion HD Cloning kit (TAKARABIO, Inc., Shiga, Japan). The resultant plasmid was named pHLA-cadA-blc-Tfu.

\section{Transformation of E. coli}

Transformation of $E$. coli was carried out by electroporation with a $1350 \mathrm{kV}, 600 \Omega, 10 \mu \mathrm{F}$ electric pulse in a $0.1 \mathrm{~cm}$ cuvette using a Gene Pulser (Bio-Rad Laboratories, Hercules, CA, USA). E. coli JCM20137 harboring pHLA-cadA, pHLA-blc-Tfu or pHLA-cadA-blc-Tfu was designated Jm-cadA, Jm-blc-Tfu and Jm-cadA-blc-Tfu, respectively.

\section{Western blotting}

E. coli cells cultured at $37^{\circ} \mathrm{C}$ for $24 \mathrm{~h}$ were analyzed by sodium dodecyl sulfate-polyacrylamide gel electrophoresis (SDS-PAGE). Proteins were analyzed by SDS-PAGE using an SDS-polyacrylamide gel (10\%; w/v). Dual color prestained Precision Plus protein standards (Bio-Rad Laboratories, Richmond, CA, USA) were used as molecular weight markers. The proteins were electroblotted onto a polyvinylidene difuluoride membrane (Millipore, Boston, MA, USA) and allowed to react with primary mouse anti-FLAG (Sigma) and secondary goat antimouse immunoglobulin G alkaline-phosphatase-conjugated antibodies (Promega Co., Madison, WI, USA). The membrane was then stained with nitroblue tetrazolium (NBT; Promega) and 5-bromo-4chloro-3-indolylphosphate (BCIP; Promega) according to the manufacturer's protocol.

\section{Measurement of BGL activity}

Recombinant strains were cultured in $5 \mathrm{ml}$ of $\mathrm{LB}$ medium containing $100 \mathrm{mg} / \mathrm{l}$ ampicillin. After incubation at $37^{\circ} \mathrm{C}$ for $24 \mathrm{~h}$, the culture $(2.5 \%$; v/v) was used to inoculate $5 \mathrm{ml}$ of $\mathrm{R} / 2$ medium containing $40 \mathrm{~g} / \mathrm{l}$ cellobiose and $100 \mathrm{mg} / \mathrm{l}$ ampicillin, then the cells were grown at $37^{\circ} \mathrm{C}$ for $48 \mathrm{~h}$. The BGL activity on the cell surface was quantitatively evaluated using $p$-nitrophenyl- $\beta$ - $D$ glucopyranoside (pNPG; Nacalai Tesque, Inc., Kyoto, 
Table 1 Strains, plasmids, and primers used in this study

\begin{tabular}{|c|c|c|}
\hline Strain, plasmid, or primer & Relevant phenotype, description, or sequence $\left(5^{\prime}-3^{\prime}\right)$ & Source or reference \\
\hline \multicolumn{3}{|l|}{ Strains } \\
\hline \multicolumn{3}{|l|}{ Escherichia coli } \\
\hline NovaBlue & $\begin{array}{l}\text { endA1 hsdR17 (rK12- mK12+) supE44 thi-1 recA1 gyrA96 relA1 } \\
\left.\text { lac[F' proAB }{ }^{+} \text {lacl }{ }^{9} Z \Delta M 15:: T n 10\left(\text { Tet }^{\prime}\right)\right] \text {; host for DNA manipulation }\end{array}$ & Novagen \\
\hline \multirow[t]{2}{*}{ JCM20137 } & & National Institute of Genetics, Japan \\
\hline & & Japan Collection of Microorganisms, RIKEN BRC \\
\hline Jm-cadA & JCM20137 strain harboring pHLA-cadA vector & This study \\
\hline Jm-blc-Tfu & JCM20137 strain harboring pHLA-blc-Tfu vector & This study \\
\hline Jm-cadA-blc-Tfu & JCM20137 strain harboring pHLA-cadA-blc-Tfu vector & This study \\
\hline \multicolumn{3}{|l|}{ Genomic DNA } \\
\hline Thermobifida fusca YX & ATCC BAA-629D-5 & ATCC \\
\hline \multicolumn{3}{|l|}{ Plasmids } \\
\hline $\mathrm{pHLA}$ & $\begin{array}{l}\text { Cell surface display vector containing pgsA gene under HCE } \\
\text { promoter control, ampicillin resistance marker }\end{array}$ & Tanaka et al. 2011 \\
\hline pHLA-cadA & Vector for CadA expression & This study \\
\hline pHLA-blc-Tfu & $\begin{array}{l}\text { Vector for BGL (Tfu0937 from T. fusca) expression using blc } \\
\text { anchor protein }\end{array}$ & Tanaka et al. 2011 \\
\hline pHLA-cadA-blc-Tfu & $\begin{array}{l}\text { Vector for CadA and BGL (Tfu0937 from T. fusca) coexpression } \\
\text { using blc anchor protein }\end{array}$ & This study \\
\hline \multicolumn{3}{|l|}{ Oligonucleotide primers } \\
\hline CadA_F & agcagatctgatgaacgttattgcaatattgaatcacatggggg & \\
\hline CadA-FLAG_R & ccagtcgacttatttatcgtcatcatctttataatcttttttgctttcttc & \\
\hline HCE-blc-Tfu-term_F & cgccgtagcgccgatggtagtgtggggtctccccatgcgag & \\
\hline HCE-blc-Tfu-term_R & ggagagatcgcggccgccatggggtcaggtgggaccaccgcgctac & \\
\hline pHLA-cadA_F & gcggecgcgatctctccttcacagattcccaatctcttgtt & \\
\hline pHLA-cadA_R & ctcgcatggggagaccccacactaccatcggcgctacggcg & \\
\hline
\end{tabular}

Japan) as a substrate. One unit of $\beta$-glucosidase activity was defined as the amount of enzyme producing $1 \mu \mathrm{mol} / \mathrm{min}$ p-nitrophenol at $37^{\circ} \mathrm{C}$ and $\mathrm{pH} 5.0$.

\section{Fermentation experiments}

Cadaverine fermentation by recombinant $E$. coli was performed in test tubes with a $5 \mathrm{ml}$ working volume. $\mathrm{R} / 2$ medium containing $40 \mathrm{~g} / \mathrm{l}$ glucose or $40 \mathrm{~g} / \mathrm{l}$ cellobiose as the sole carbon source was used. $E$. coli cells grown on LB medium containing $100 \mathrm{mg} / \mathrm{l}$ ampicillin at $37^{\circ} \mathrm{C}$ for $24 \mathrm{~h}$ at $180 \mathrm{rpm}$ were used to inoculate $5 \mathrm{ml}$ of $\mathrm{R} / 2$ containing $40 \mathrm{~g} / \mathrm{l}$ glucose or cellobiose at $37^{\circ} \mathrm{C}, 180 \mathrm{rpm}$. The initial OD600 was adjusted to 0.1 . The initial $\mathrm{pH}$ of the medium was 6.8; the $\mathrm{pH}$ was not controlled during fermentation.

\section{Analytical methods}

Throughout the $48 \mathrm{~h}$ of cultivation, BGL activities were measured as described above. Cell growth was monitored by measuring the OD600 with an UVmini-1240 UV-Vis spectrophotometer (Shimadzu, Kyoto, Japan). Cellobiose and glucose in culture supertnatants were analyzed using a Prominence HPLC System (Shimadzu) equipped with a SPR-Pb column $(0.5 \mu \mathrm{m}, 250 \times 4.0 \mathrm{~mm}$ I.D.; Shimadzu). Water was used as the mobile phase at a flow rate of $0.6 \mathrm{ml} / \mathrm{min}$, and the column was maintained at $80^{\circ} \mathrm{C}$. The peak elution profile was monitored using a refractive index detector. The cadaverine concentration in the supernatant was analyzed using a TSKgel Polyaminepak (7 $\mu \mathrm{m}, 4.6 \times 50 \mathrm{~mm}$ I.D.; TOSOH CORPORATION, Yamaguchi, Japan) using a Prominence Amino Acid Analyzer System (Shimadzu) after derivation with orthophthalaldehyde, according to manufacturer's procedure. The mobile phase was a step gradient of a sodium citrate solution, $\mathrm{pH} 5.28$, at a flow rate of $0.4 \mathrm{ml} /$ min, and the column was maintained at $50^{\circ} \mathrm{C}$, also according to manufacturer's procedure.

\section{Results}

\section{Expression of CadA in E. coli}

Strains evaluated in this study are summarized in Table 1. Lysine decarboxylase (CadA) expression in E. coli is one of the important factors for cadaverine production. To confirm the expression of CadA in E. coli, intracellular 
fractions of E. coli cells were analyzed by Western blot analysis after $24 \mathrm{~h}$ of cultivation using glucose as a carbon source. Intracellular extracts of Jm-cadA and Jm-cadA-blc-Tfu showed a clear band corresponding to CadA-FLAG (82 kDa) (Figure 1, lanes 1 and 3). In contrast, no band was observed from extracts of Jmblc-Tfu (Figure 1, lane 2). These results indicate the successful intracellular expression of CadA in Jm-cadA and Jm-cadA-blc-Tfu.

\section{BGL expression using $E$. coli as a host}

BGL expression is needed for the efficient utilization of cellobiose. BGL activity on the cell surface of the BGLexpressing strains was quantitatively evaluated using pNPG as a substrate. As shown in Table 2, both of Jm-blcTfu and Jm-cadA-blc-Tfu showed higher BGL activity when grown on cellobiose (0.70 U/OD600/l of Jm-blc-Tfu and $0.74 \mathrm{U} / \mathrm{OD} 600 / \mathrm{l}$ of Jm-cadA-blc-Tfu) compared to those grown on glucose $(0.18 \mathrm{U} / \mathrm{OD} 600 / \mathrm{l}$ of Jm-blc-Tfu and $0.13 \mathrm{U} / \mathrm{OD} 600 / \mathrm{l}$ of Jm-cadA-blc-Tfu). No activity was detected on Jm-cadA cells. These results indicate that BGL was successfully expressed on the cell surface of Jmblc-Tfu and Jm-cadA-blc-Tfu cells and retained its enzymatic function through the Blc anchor protein.

\section{Cadaverine fermentation from glucose}

We then evaluated cadaverine production by Jm-cadA, Im-blc-Tfu and Jm-cadA-blc-Tfu grown in R/2 medium using glucose as the sole carbon source. Figure 2 shows

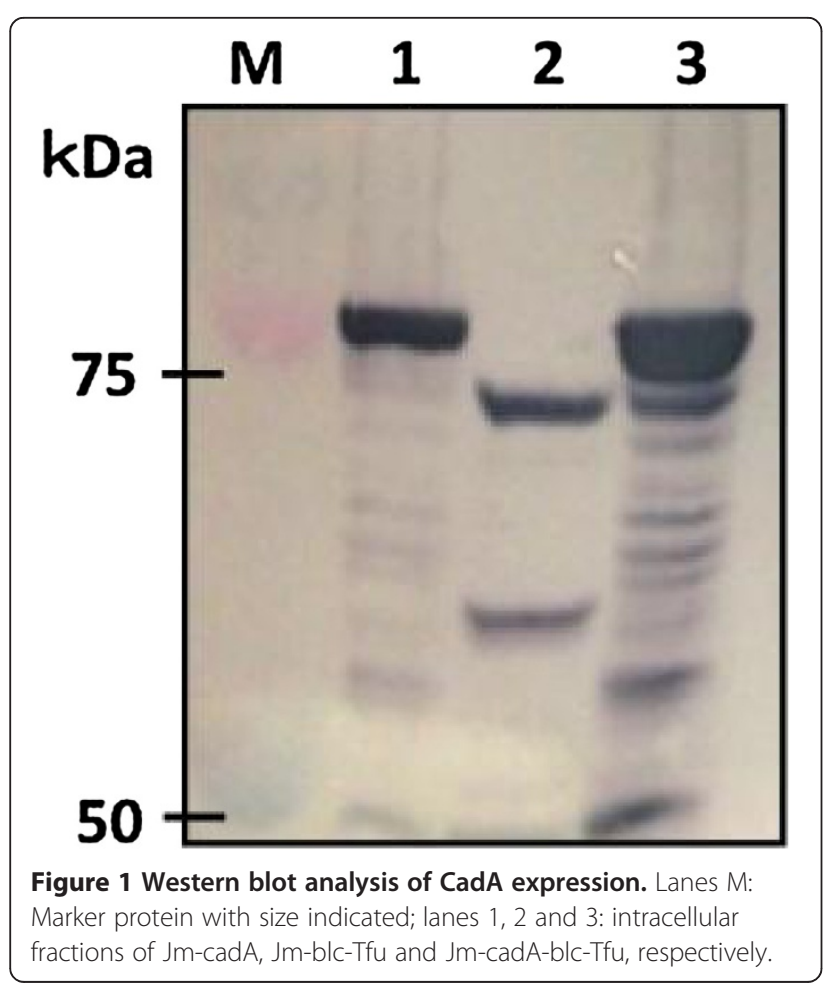

Table 2 BGL activities using Jm-cadA, Jm-blc-Tfu and $\mathrm{Jm}$-cadA-blc-Tfu grown on glucose $(40 \mathrm{~g} / \mathrm{l})$ or cellobiose $(40 \mathrm{~g} / \mathrm{l})$ as a carbon source

\begin{tabular}{lll}
\hline Strains & $\begin{array}{l}\text { BGL activity grown on } \\
\text { glucose (U/OD600/I) }\end{array}$ & $\begin{array}{l}\text { BGL activity grown on } \\
\text { cellobiose (U/OD600/I) }\end{array}$ \\
\hline Jm-cadA & Not detected & Not growth \\
Jm-blc-Tfu & $0.18 \pm 0.12$ & $0.70 \pm 0.03$ \\
Jm-cadA-blc-Tfu & $0.13 \pm 0.08$ & $0.74 \pm 0.07$ \\
\hline
\end{tabular}

the cell growth, glucose consumption and cadaverine concentration. As shown in Figure 3a, all strains showed similar growth, with OD600 values after $12 \mathrm{~h}$ cultivation of 5.4 (Jm-cadA), 5.9 (Jm-blc-Tfu) and 5.7 (Jm-CadAblc-Tfu). The amount of consumed glucose after $24 \mathrm{~h}$ was $18.3 \mathrm{~g} / \mathrm{l}$ (Jm-cadA), $20.3 \mathrm{~g} / \mathrm{l}$ (Jm-blc-Tfu) and $20.3 \mathrm{~g} /$ 1 (Jm-CadA-blc-Tfu) (Figure 2b). As shown in Figure 2c, cadaverine production by the CadA-expressing strains was higher than by the control strain. The cadaverine concentration by Jm-cadA-blc-Tfu was $3.3 \mathrm{mM}$ at $48 \mathrm{~h}$, $1.5 \mathrm{mM}$ by Jm-cadA and $0.1 \mathrm{mM}$ by Jm-blc-Tfu. These results show that CadA-expression in E. coli resulted in higher cadaverine production.

\section{Cadaverine fermentation from cellobiose}

We then evaluated cadaverine production using cellobiose as the sole carbon source. Figure 3 shows the cell growth, cellobiose consumption and cadaverine concentration using cellobiose as the sole carbon source in $\mathrm{R} / 2$ medium. The BGL-expressing strains, Jm-blc-Tfu and Jm-cadA-blc-Tfu, grew on cellobiose, providing OD600 values after $48 \mathrm{~h}$ cultivation of 10.1 (Jm-blc-Tfu) and 8.0 (Jm-cadA-blc-Tfu). Strain Jm-cadA, which does not express BGL, did not grow on cellobiose.

As shown in Figure 3b, BGL-expressing strains started consuming cellobiose after 24 h cultivation, with $28.2 \mathrm{~g} / \mathrm{l}$ (Jm-blc-Tfu) and $28.0 \mathrm{~g} / \mathrm{l}$ (Jm-cadA-blc-Tfu) cellobiose consumed after $48 \mathrm{~h}$. Glucose was not detected in the culture medium during cultivation (data not shown).

The cadaverine concentration produced from cellobiose is shown in Figure 3c. No cadaverine was detected in the culture medium of Jm-cadA since it did not grow on cellobiose. In the case of Jm-blc-Tfu, only $0.1 \mathrm{mM}$ of cadaverine was detected after $48 \mathrm{~h}$, even though the strain grew on cellobiose (Figure $3 \mathrm{~b}$ ). In contrast, strain Jm-cadA-blc-Tfu produced $6.1 \mathrm{mM}$ cadaverine after $48 \mathrm{~h}$. This amount of cadaverine production is higher than that observed after $48 \mathrm{~h}$ of glucose fermentation by Jm-cadA-blc-Tfu.

\section{Discussion}

The aim of this study was the direct production of cadaverine from cellobiose using $E$. coli expressing BGL and CadA. We previously reported the direct utilization 


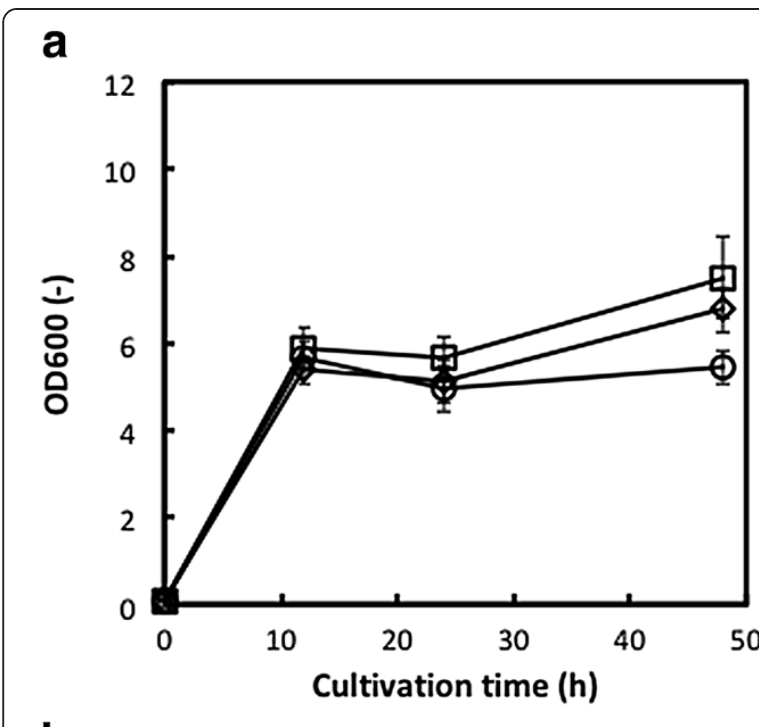

b

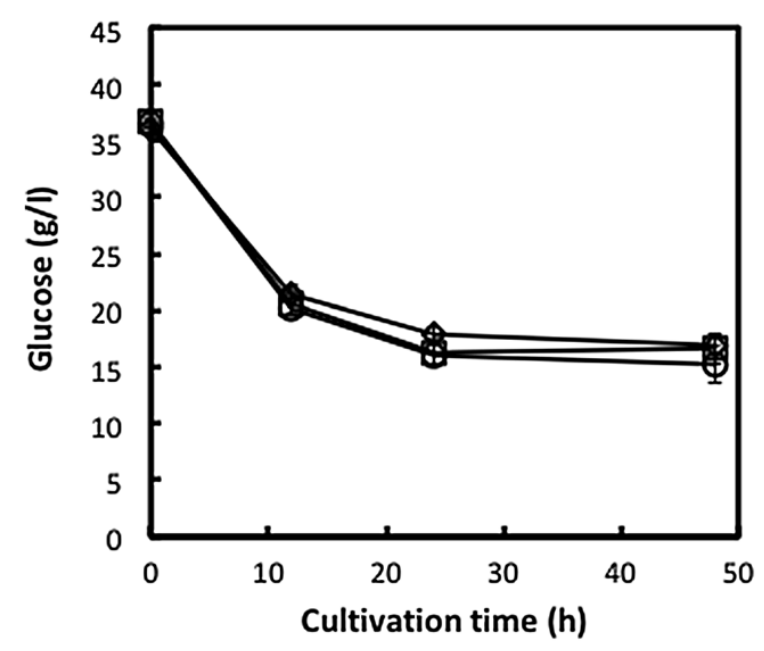

C

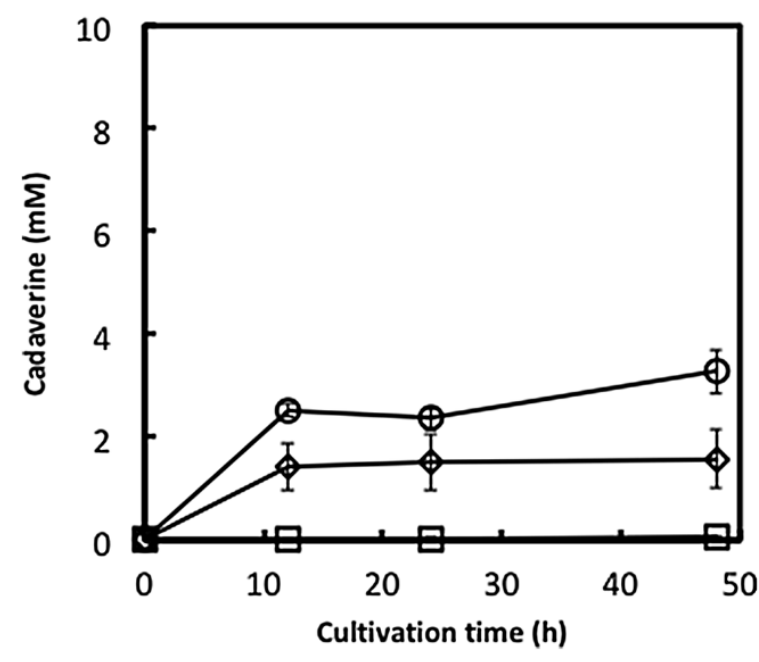

Figure 2 Cadaveine fermentation experiments with glucose as the sole carbon source using Jm-cadA (diamonds), Jm-blc-Tfu (squares) and Jm-cadA-blc-Tfu (circles). (a), Cell growth measured by OD600, (b), glucose concentration and (c), cadaverine concentration. Data points represent means and standard deviation of three independent experiments.

of cellobiose using E. coli displaying BGL (Tanaka et al. 2011). Here, we engineered $E$. coli to produce cadaverine efficiency from cellobiose. This is the first report of cadaverine production using cellobiose as the sole carbon source by engineered $E$. coli.

Most wild-type Escherichia coli cells are unable to use cellobiose due to the cryptic nature of the cellobioseutilizing operons such as bgl and cel (Parker and Hall 1990). There are two pathways for cellobiose utilization in E. coli. One is the direct import of cellobiose through the PTS (PEP-dependent phosphotransferase system). Indeed, it was reported that a cellobiose-positive phenotype could be easily obtained by prolonged cultivation using cellobiose as the sole carbon source (Hall and Betts 1987; Keyhani and Roseman 1997; Parker and Hall 1990). Efforts to engineer $E$. coli to use cellobiose are of interest as commercial cellulase cocktails lack sufficient beta-glucosidase activity, which makes supplementation with beta-glucosidase necessary (Xin et al. 1993). Alternatively, expressing the cel operon from Klebsiella oxytoca in E. coli allows cellobiose to be transported through a phosphotransferase system to yield intracellular cellobiose-phosphate, which is subsequently cleaved by another operon-encoded enzyme, phospho-beta-glucosidase, to yield glucose-6phosphate and glucose (Moniruzzaman et al. 1997).

The other approach is the heterologous expression of BGL and the conversion of cellobiose to glucose. Although the degradation of cellobiose is the rate-limiting step, and Tfu0937 described in this study currently exhibits insufficient BGL activity, the expression of a suitable BGL and optimization of fermentation conditions will improve productivity. The expression of betaglucosidase and its display on the cell surface has been attempted and resulted in some reduction in the need for BGL (Ha et al. 2011).

In this study, we constructed BGL- and CadAexpressing E. coli. The BGL activity of Jm-blc-Tfu and Jm-CadA-blc-Tfu grown on cellobiose was higher than those grown on glucose (Table 2). Although all E.coli strains can be grown on glucose, the suitable E.coli strain (i.e. having high BGL activity) was selected during the cultivation using cellobiose as a carbon source. No glucose was detected in the culture medium during fermentation, suggesting that the rate-limiting step was cellobiose hydrolysis; if this is the case, then improving BGL activity on the cell surface would lead to cadaverine 


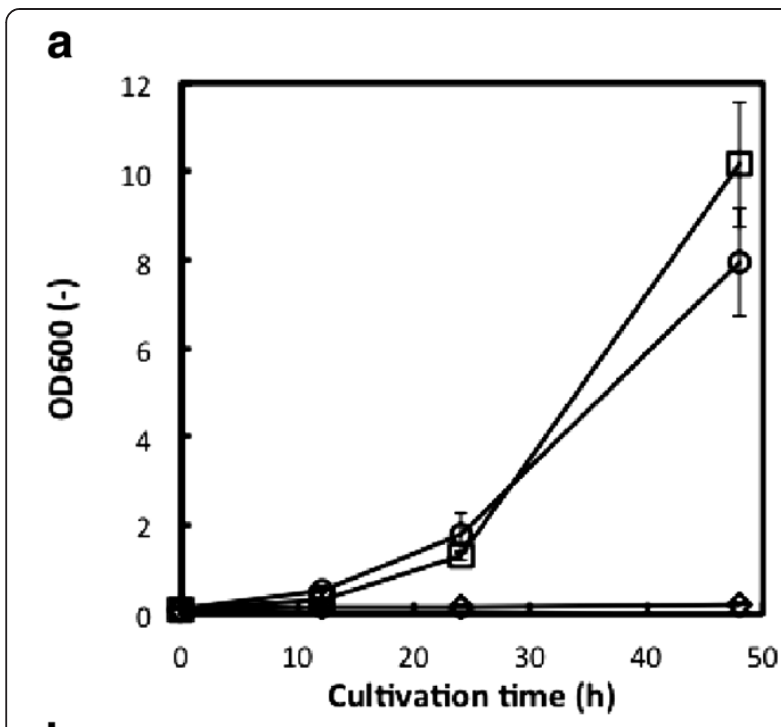

b

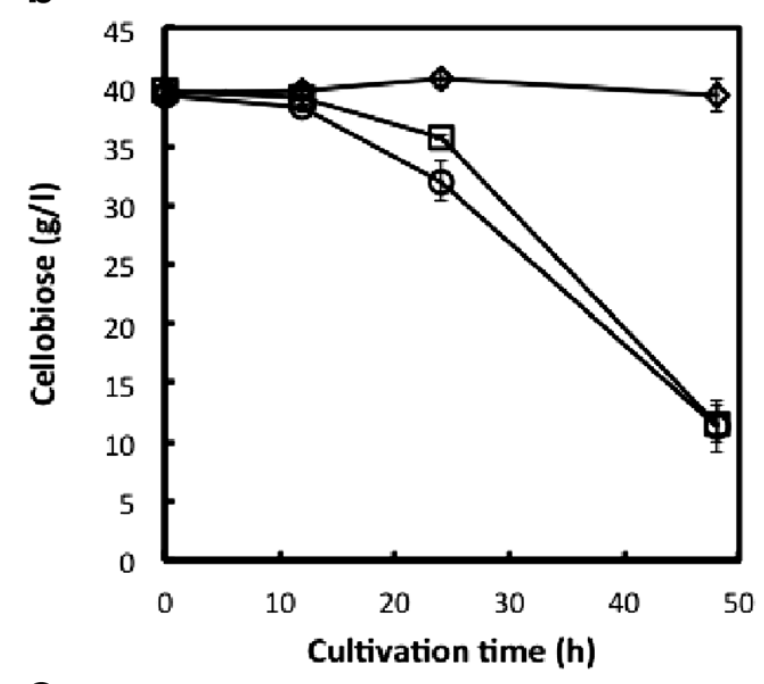

C

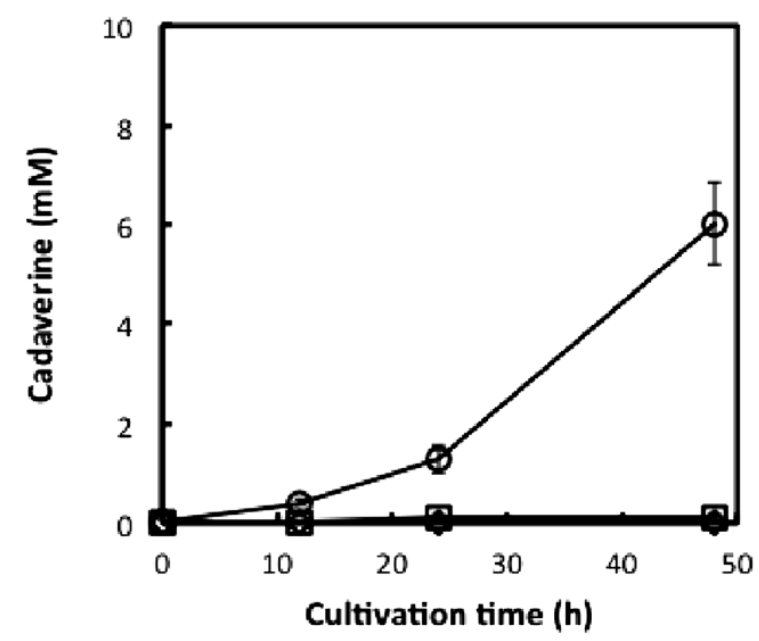

Figure 3 Cadaveine fermentation experiments with cellobiose as the sole carbon source using Jm-cadA (diamonds), Jm-blcTfu (squares) and Jm-cadA-blc-Tfu (circles). (a), Cell growth measured by OD600, (b), cellobiose concentration and (c), cadaverine concentration. Data points represent means and standard deviation of three independent experiments.

production. That the growth rate with glucose as the carbon source was higher than when cellobiose was the carbon source also suggests that increasing the glucose concentration produced by BGL from cellobiose would improve cadaverine production.

CadA expression in E. coli increased the amount of cadaverine produced, from $0.1 \mathrm{mM}$ up to $3.3 \mathrm{mM}$, using glucose as the carbon source (Figure 2). The utility of CadA overexpression for cadaverine production from glucose has been previously demonstrated; for example, $12.8 \mathrm{mM}$ in E. coli (Qian et al. 2011) and $50 \mathrm{mM}$ in C. glutamicum (Tateno et al. 2009). Metabolic engineering of the cadaverine production pathway is also a promising approach for increasing cadaverine production. Na et al. (2013) demonstrated cadaverine production using metabolic pathway engineered E. coli and obtained up to $21.1 \mathrm{mM}$ cadaverine. Pathway engineering of C. glutamicum also improved cadaverine productivity (Buschke et al. 2013a, b; Kind et al. 2011). In this study, we demonstrated direct cadaverine production from cellobiose using a BGLdisplaying and cadA-expressing E. coli strain. Interestingly, when cellobiose was used as the sole carbon source, the OD600 values were higher than when glucose was used as the sole carbon source (Figures 2a and 3a). Additionally, the amount of cadaverine produced from cellobiose was higher than from glucose (Figures $2 \mathrm{c}$ and $3 \mathrm{c}$ ), although the growth rate, sugar consumption rate and cadaverine production rate from cellobiose were lower than when the cells were grown on glucose. It is possible that the sugar concentration around the cells may affect metabolism; if so, than providing controlled amounts of glucose to the culture may help improve cadaverine production. However, sugar consumption and cadaverine production were prevented after $24 \mathrm{~h}$ fermentation (Figures 2 and 3 ), which was possibly caused by low pH (about 4.0 after 48 h cultivation). Controlling $\mathrm{pH}$ during fermentation may be improved cadaverine productivity, as well as metabolic engineering.

In conclusion, we have demonstrated the direct production of cadaverine from cellobiose using BGL-displaying and CadA-expressing E. coli. Active BGL Tfu0937 was displayed on the cell surface and direct cadaverine fermentation from cellobiose was demonstrated. Our results indicate that cellobiose is a more suitable substrate for $E$. coli expressing BGL than glucose in some cases. For the direct utilization of cellulose, other kinds of cellulases, such as EG and $\mathrm{CBH}$, are required. To address this, we are currently 
constructing E. coli coexpressing cellulases for the direct and efficient production of cadaverine from cellulose.

\section{Competing interests}

The authors declare that they have no competing interests.

\section{Acknowledgments}

This work was supported in part by Grant-in-Aid for Scientific Research (B) and by Special Coordination Funds for Promoting Science and Technology, Creation of Innovation Centers for Advanced Interdisciplinary Research Areas (Innovative Bioproduction Kobe), MEXT, Japan.

\section{Received: 3 September 2013 Accepted: 31 October 2013}

Published: 8 November 2013

\section{References}

Atsumi S, Hanai T, Liao JC (2008) Non-fermentative pathways for synthesis of branched-chain higher alcohols as biofuels. Nature 451:86-89

Buschke N, Schäfer R, Becker J, Wittmann C (2013a) Metabolic engineering of industrial platform microorganisms for biorefinery applications-optimization of substrate spectrum and process robustness by rational and evolutive strategies. Bioresour Technol 135:544-554

Buschke N, Becker J, Schäfer R, Kiefer P, Biedendieck R, Wittmann C (2013b) Systems metabolic engineering of xylose-utilizing Corynebacterium glutamicum for production of 1,5-diaminopentane. Biotechnol J 8:557-570

Ha SJ, Galazka JM, Kim SR, Choi JH, Yang X, Seo JH, Glass NL, Cate JH, Jin YS (2011) Engineered Saccharomyces cerevisiae capable of simultaneous cellobiose and xylose fermentation. Proc Natl Acad Sci U S A 108:504-509

Hall BG, Betts PW (1987) Cryptic genes for cellobiose utilization in natural isolates of Escherichia coli. Genetics 115:431-439

Keyhani NO, Roseman S (1997) Wild-type Escherichia coli grows on the chitin disaccharide, N, N =-diacetylchitobiose, by expressing the cel operon. Proc Natl Acad Sci 94:14367-14371

Kind S, Jeong WK, der Schro H, Wittmann C (2010) Systems-wide metabolic pathway engineering in Corynebacterium glutamicum for bio-based production of diaminopentane. Metab Eng 12:341-351

Kind S, Kreye S, Wittmann C (2011) Metabolic engineering of cellular transport for overproduction of the platform chemical 1,5-diaminopentane in Corynebacterium glutamicum. Metab Eng 13:217-617

Lee SY, Chang HN (1993) High cell density cultivation of Escherichia coli W using sucrose as a carbon source. Biotechnol Lett 15:971-974

Lee SY, Choi JH, Xu Z (2003) Microbial cell-surface display. Trends Biotechnol 21(1):45-52

Lee KH, Park JH, Kim TY, Kim HU, Lee SY (2007) Systems metabolic engineering of Escherichia coli for L-threonine production. Mol Syst Biol 3:149

Mimitsuka T, Sawai H, Hatsu M, Yamada K (2007) Metabolic engineering of Corynebacterium glutamicum for cadaverine fermentation. Biosci Biotechnol Biochem 71:2130-2135

Moniruzzaman M, Lai XK, York SW, Ingram LO (1997) Isolation and molecular characterization of high-performance cellobiose-fermenting spontaneous mutants of ethanologenic Escherichia coli KO11 containing the Klebsiella oxytoca cas AB operon. Appl Environ Microbiol 63:4633-4637

Na D, Yoo SM, Chung H, Park H, Park JH, Lee SY (2013) Metabolic engineering of Escherichia coli using synthetic small regulatory RNAs. Nat Biotechnol 31 (2): 170-174

Narita J, Okano K, Tateno T, Tanino T, Sewaki T, Sung MH, Fukuda H, Kondo A (2006) Display of active enzymes on the cell surface of Escherichia coli using PgsA anchor protein and their application to bioconversion. Appl Microbiol Biotechnol 70(5):564-572

Park JH, Lee KH, Kim TY, Lee SY (2007) Metabolic engineering of Escherichia coli for the production of L-valine based on transcriptome analysis and in silico gene knockout simulation. Proc Natl Acad Sci 104:7797-7802

Park JH, Lee SY, Kim TY, Kim HU (2008) Application of systems biology for bioprocess development. Trends Biotechnol 26:404-412

Parker LL, Hall BG (1990) Mechanisms of activation of the cryptic cel operon of Escherichia coli K12. Genetics 124:473-482

Qian ZG, Xia XX, Lee SY (2009) Metabolic engineering of Escherichia coli for the production of putrescine: A four carbon diamine. Biotechnol Bioeng 104:651-662
Qian ZG, Xia XX, Lee SY (2011) Metabolic engineering of Escherichia coli for the production of cadaverine: a five carbon diamine. Biotechnol Bioeng 108(1):93-103

Sánchez OJ, Cardona CA (2008) Trends in biotechnological production of fue ethanol from different feedstocks. Bioresour Technol 99(13):5270-5295

Soksawatmaekhin W, Kuraishi A, Sakata K, Kashiwagi K, Igarashi K (2004) Excretion and uptake of cadaverine by $\mathrm{CadB}$ and its physiological functions in Escherichia coli. Mol Microbiol 51:1401-1412

Soma Y, Inokuma K, Tanaka T, Ogino C, Kondo A, Okamoto M, Hanai T (2012) Direct isopropanol production from cellobiose by engineered Escherichia coli using a synthetic pathway and a cell surface display system. J Biosci Bioeng 114:80-85

Steen EJ, Kang Y, Bokinsky G, Hu Z, Schirmer A, McClure A, Del Cardayre SB, Keasling JD (2010) Microbial production of fatty-acid- derived fuels and chemicals from plant biomass. Nature 463:559-562

Tanaka T, Kawabata H, Ogino C, Kondo A (2011) Creation of a cellooligosaccharide-assimilating Escherichia coli strain by displaying active $\beta$-glucosidase on the cell surface via a novel anchor protein. Appl Environ Microbiol 77(17):6265-6270

Tateno T, Okada Y, Tsuchidate T, Tanaka T, Fukuda H, Kondo A (2009) Direct production of cadaverine from soluble starch using Corynebacterium glutamicum coexpressing a-amylase and lysine decarboxylase. Appl Microbiol Biotechnol 82(1):115-121

Tyo KE, Ajikumar PK, Stephanopoulos G (2009) Stabilized gene duplication enables long-term selection-free heterologous pathway expression. Nat Biotechnol 27:760-765

Xin Z, Yinbo Q, Peiji G (1993) Acceleration of ethanol-production from paper-mill waste fiber by supplementation with $\beta$-glucosidase. Enzyme Microb Technol 15:62-65

doi:10.1186/2191-0855-3-67

Cite this article as: Ikeda et al:: Direct cadaverine production from cellobiose using $\beta$-glucosidase displaying Escherichia coli. AMB Express 2013 3:67.

\section{Submit your manuscript to a SpringerOpen ${ }^{\odot}$ journal and benefit from:}

- Convenient online submission

- Rigorous peer review

- Immediate publication on acceptance

- Open access: articles freely available online

- High visibility within the field

- Retaining the copyright to your article

Submit your next manuscript at $>$ springeropen.com 